\title{
PERIODIC MINIMAL SURFACES IN SEMIDIRECT PRODUCTS
}

\author{
ANA MENEZES \\ (Received 14 January 2013; accepted 14 January 2013; first published online 15 October 2013) \\ Communicated by G. Willis
}

\begin{abstract}
In this paper we prove the existence of complete minimal surfaces in some metric semidirect products. These surfaces are similar to the doubly and singly periodic Scherk minimal surfaces in $\mathbb{R}^{3}$. In particular, we obtain these surfaces in the Heisenberg space with its canonical metric, and in $\mathrm{Sol}_{3}$ with a oneparameter family of nonisometric metrics.
\end{abstract}

2010 Mathematics subject classification: primary 53C42.

Keywords and phrases: semidirect products, minimal surfaces.

\section{Introduction}

In this paper we construct examples of periodic minimal surfaces in some semidirect products $\mathbb{R}^{2} \rtimes_{A} \mathbb{R}$, depending on the matrix $A$. By 'periodic surface' we mean a properly embedded surface invariant with respect to a nontrivial discrete group of isometries.

One of the simplest examples of a semidirect product is $\mathbb{H}^{2} \times \mathbb{R}=\mathbb{R}^{2} \rtimes_{A} \mathbb{R}$, when we take $A=\left(\begin{array}{ll}1 & 0 \\ 0 & 0\end{array}\right)$. In this space, Mazet et al. [2] proved some results about periodic constant mean curvature surfaces and constructed examples of such surfaces. One of their methods is to solve a Plateau problem for a certain contour. In [5], using a similar technique, Rosenberg constructed examples of complete minimal surfaces in $M^{2} \times \mathbb{R}$, where $M$ is either the 2 -sphere or a complete Riemannian surface with nonnegative curvature or the hyperbolic plane.

Meeks et al. [3] have proved results concerning the geometry of solutions to Plateau type problems in metric semidirect products $\mathbb{R}^{2} \rtimes_{A} \mathbb{R}$, when there is some geometric constraint on the boundary values of the solution (see Theorem 2.5).

The first example that we construct is a complete periodic minimal surface similar to the doubly periodic Scherk minimal surface in $\mathbb{R}^{3}$. It is invariant with respect to two

The author was financially supported by CNPq-Brazil and IMPA.

(C) 2013 Australian Mathematical Publishing Association Inc. 1446-7887/2013 \$16.00 
translations that commute and is a four-punctured sphere in the quotient of $\mathbb{R}^{2} \rtimes_{A} \mathbb{R}$ by the group of isometries generated by the two translations. In the final section we obtain a complete periodic minimal surface analogous to the singly periodic Scherk minimal surface in $\mathbb{R}^{3}$.

These surfaces are obtained by solving the Plateau problem for a geodesic polygonal contour $\Gamma$ (it uses a result by Meeks et al. [3] about the geometry of solutions to the Plateau problem in semidirect products), and letting some sides of $\Gamma$ tend to infinity in length, so that the associated Plateau solutions all pass through a fixed compact region (this will be assured by the existence of minimal annuli playing the role of barriers). Then a subsequence of the Plateau solutions will converge to a minimal surface bounded by a geodesic polygon with edges of infinite length. We complete this surface by symmetry across the edges. The whole construction requires precise geometric control and uses curvature estimates for stable minimal surfaces.

These results are obtained for semidirect products $\mathbb{R}^{2} \rtimes_{A} \mathbb{R}$ where $A=\left(\begin{array}{ll}0 & b \\ c & 0\end{array}\right)$. For example, we obtain periodic minimal surfaces in the Heisenberg space, when $A=\left(\begin{array}{ll}0 & 1 \\ 0 & 0\end{array}\right)$, and in $\mathrm{Sol}_{3}$, when $A=\left(\begin{array}{ll}0 & 1 \\ 1 & 0\end{array}\right)$, with their well-known Riemannian metrics. When we consider the one-parameter family of matrices $A(c)=\left(\begin{array}{cc}0 & c \\ 1 / c & 0\end{array}\right), c \geq 1$, we get a oneparameter family of metrics in $\mathrm{Sol}_{3}$ which are not isometric.

\section{Preliminary results}

Generalizing direct products, a semidirect product is a particular way in which a group can be constructed from two subgroups, one of which is a normal subgroup. As a set, it is the cartesian product of the two subgroups but with a particular multiplication operation.

In our case, the normal subgroup is $\mathbb{R}^{2}$ and the other subgroup is $\mathbb{R}$. Given a matrix $A \in \mathcal{M}_{2}(\mathbb{R})$, we can consider the semidirect product $\mathbb{R}^{2} \rtimes_{A} \mathbb{R}$, where the group operation is given by

$$
\left(p_{1}, z_{1}\right) *\left(p_{2}, z_{2}\right)=\left(p_{1}+e^{z_{1} A} p_{2}, z_{1}+z_{2}\right), \quad p_{1}, p_{2} \in \mathbb{R}^{2}, \quad z_{1}, z_{2} \in \mathbb{R}
$$

and

$$
A=\left(\begin{array}{ll}
a & b \\
c & d
\end{array}\right) \in \mathcal{M}_{2}(\mathbb{R}) .
$$

We choose coordinates $(x, y) \in \mathbb{R}^{2}, z \in \mathbb{R}$. Then $\partial_{x}=\partial / \partial x, \partial_{y}, \partial_{z}$ is a parallelization of $G=\mathbb{R}^{2} \rtimes_{A} \mathbb{R}$. Taking derivatives at $t=0$ in (2.1) of the left multiplication by $(t, 0,0) \in G$ (respectively by $(0, t, 0),(0,0, t))$, we obtain the following basis $\left\{F_{1}, F_{2}, F_{3}\right\}$ of the right invariant vector fields on $G$ :

$$
F_{1}=\partial_{x}, \quad F_{2}=\partial_{y}, \quad F_{3}=(a x+b y) \partial_{x}+(c x+d y) \partial_{y}+\partial_{z} .
$$

Analogously, if we take derivatives at $t=0$ in (2.1) of the right multiplication by $(t, 0,0) \in G$ (respectively by $(0, t, 0),(0,0, t))$, we obtain the following basis $\left\{E_{1}, E_{2}, E_{3}\right\}$ of the Lie algebra of $G$ :

$$
E_{1}=a_{11}(z) \partial_{x}+a_{21}(z) \partial_{y}, \quad E_{2}=a_{12}(z) \partial_{x}+a_{22}(z) \partial_{y}, \quad E_{3}=\partial_{z}
$$


where we have denoted

$$
e^{z A}=\left(\begin{array}{ll}
a_{11}(z) & a_{12}(z) \\
a_{21}(z) & a_{22}(z)
\end{array}\right) .
$$

We define the canonical left invariant metric on $\mathbb{R}^{2} \rtimes_{A} \mathbb{R}$, denoted by $\langle$, $\rangle$, to be that one for which the left invariant basis $\left\{E_{1}, E_{2}, E_{3}\right\}$ is orthonormal.

The Riemannian connection $\nabla$ for the canonical left invariant metric of $\mathbb{R}^{2} \rtimes_{A} \mathbb{R}$ in this frame is expressed as:

$$
\begin{array}{r}
\nabla_{E_{1}} E_{1}=a E_{3}, \quad \nabla_{E_{1}} E_{2}=\frac{b+c}{2} E_{3}, \quad \nabla_{E_{1}} E_{3}=-a E_{1}-\frac{b+c}{2} E_{2}, \\
\nabla_{E_{2}} E_{1}=\frac{b+c}{2} E_{3}, \quad \nabla_{E_{2}} E_{2}=d E_{3}, \quad \nabla_{E_{2}} E_{3}=-\frac{b+c}{2} E_{1}-d E_{2}, \\
\nabla_{E_{3}} E_{1}=\frac{c-b}{2} E_{2}, \quad \nabla_{E_{3}} E_{2}=\frac{b-c}{2} E_{1}, \quad \nabla_{E_{3}} E_{3}=0 .
\end{array}
$$

In particular, for every $\left(x_{0}, y_{0}\right) \in \mathbb{R}^{2}, \gamma(z)=\left(x_{0}, y_{0}, z\right)$ is a geodesic in $G$.

Remark 2.1. Since $\left[E_{1}, E_{2}\right]=0$, we have for all $z$ that $\mathbb{R}^{2} \rtimes_{A}\{z\}$ is flat and the horizontal straight lines are geodesics. Moreover, the mean curvature of $\mathbb{R}^{2} \rtimes_{A}\{z\}$ with respect to the unit normal vector field $E_{3}$ is the constant $H=\operatorname{tr}(A) / 2$.

The change from the orthonormal basis $\left\{E_{1}, E_{2}, E_{3}\right\}$ to the basis $\left\{\partial_{x}, \partial_{y}, \partial_{z}\right\}$ produces the following expression for the metric $\langle$,$\rangle :$

$$
\begin{aligned}
\langle,\rangle_{(x, y, z)}=[ & \left.a_{11}(-z)^{2}+a_{21}(-z)^{2}\right] d x^{2}+\left[a_{12}(-z)^{2}+a_{22}(-z)^{2}\right] d y^{2}+d z^{2} \\
& +\left[a_{11}(-z) a_{12}(-z)+a_{21}(-z) a_{22}(-z)\right](d x \otimes d y+d y \otimes d x) \\
= & e^{-2 \operatorname{tr}(A) z}\left\{\left[a_{21}(z)^{2}+a_{22}(z)^{2}\right] d x^{2}+\left[a_{11}(z)^{2}+a_{12}(z)^{2}\right] d y^{2}\right\}+d z^{2} \\
& -e^{-2 \operatorname{tr}(A) z}\left[a_{11}(z) a_{21}(z)+a_{12}(z) a_{22}(z)\right](d x \otimes d y+d y \otimes d x) .
\end{aligned}
$$

In particular, for every matrix $A \in \mathcal{M}_{2}(\mathbb{R})$, the rotation by angle $\pi$ around the vertical geodesic $\gamma(z)=\left(x_{0}, y_{0}, z\right)$ given by the map $R(x, y, z)=\left(-x+2 x_{0},-y+2 y_{0}, z\right)$ is an isometry of $\left(\mathbb{R}^{2} \rtimes_{A} \mathbb{R},\langle\rangle,\right)$ into itself.

REMARK 2.2. As we observed, the vertical lines of $\mathbb{R}^{2} \rtimes_{A} \mathbb{R}$ are geodesics of its canonical metric. For any line $l$ in $\mathbb{R}^{2} \rtimes_{A}\{0\}$ let $P_{l}$ denote the vertical plane $\{(x, y, z)$ : $(x, y, 0) \in l ; z \in \mathbb{R}\}$ containing the set of vertical lines passing through $l$. It follows that $P_{l}$ is ruled by vertical geodesics and, since rotation by angle $\pi$ around any vertical line in $P_{l}$ is an isometry that leaves $P_{l}$ invariant, $P_{l}$ has zero mean curvature.

Although the rotation by angle $\pi$ around horizontal geodesics is not always an isometry, we have the following result.

Proposition 2.3. Let $A=\left(\begin{array}{ll}0 & b \\ c & 0\end{array}\right) \in \mathcal{M}_{2}(\mathbb{R})$ and consider the horizontal geodesic $\alpha=$ $\left\{\left(x_{0}, t, 0\right): t \in \mathbb{R}\right\}$ in $\mathbb{R}^{2} \rtimes_{A}\{0\}$ parallel to the $y$-axis. Then the rotation by angle $\pi$ around $\alpha$ is an isometry of $\left(\mathbb{R}^{2} \rtimes_{A} \mathbb{R},\langle\rangle,\right)$ into itself. The same result is true for a horizontal geodesic parallel to the $x$-axis. 
Proof. The rotation by angle $\pi$ around $\alpha$ is given by the map $\phi(x, y, z)=(-x+$ $\left.2 x_{0}, y,-z\right)$, so $\phi_{x}=-\partial_{x}, \phi_{y}=\partial_{y}$ and $\phi_{z}=-\partial_{z}$.

If $A=\left(\begin{array}{ll}0 & b \\ c & 0\end{array}\right)$, then

$$
e^{z A}=\left(\begin{array}{ll}
\sum_{k=0}^{\infty} \frac{(b c)^{k} z^{2 k}}{(2 k) !} & \sum_{k=1}^{\infty} \frac{b^{k} c^{k-1} z^{2 k-1}}{(2 k-1) !} \\
\sum_{k=1}^{\infty} \frac{c^{k} b^{k-1} z^{2 k-1}}{(2 k-1) !} & \sum_{k=0}^{\infty} \frac{(b c)^{k} z^{2 k}}{(2 k) !}
\end{array}\right) .
$$

Hence, $a_{11}(z)=a_{22}(z)$ and $e^{-z A}=\left(\begin{array}{cc}a_{11}(z) & -a_{12}(z) \\ -a_{21}(z) & a_{11}(z)\end{array}\right)$. Then

$$
\begin{aligned}
\langle,\rangle_{(x, y, z)}=\{[ & \left.\left.a_{21}(z)^{2}+a_{11}(z)^{2}\right] d x^{2}+\left[a_{11}(z)^{2}+a_{12}(z)^{2}\right] d y^{2}\right\}+d z^{2} \\
- & {\left[a_{11}(z) a_{21}(z)+a_{12}(z) a_{11}(z)\right](d x \otimes d y+d y \otimes d x) }
\end{aligned}
$$

and

$$
\begin{aligned}
\langle,\rangle_{\phi(x, y, z)}=\{[ & \left.\left.a_{21}(z)^{2}+a_{11}(z)^{2}\right] d x^{2}+\left[a_{11}(z)^{2}+a_{12}(z)^{2}\right] d y^{2}\right\}+d z^{2} \\
+ & {\left[a_{11}(z) a_{21}(z)+a_{12}(z) a_{11}(z)\right](d x \otimes d y+d y \otimes d x) . }
\end{aligned}
$$

Therefore, $\left\langle\phi_{x}, \phi_{x}\right\rangle_{\phi(x, y, z)}=\left\langle\partial_{x}, \partial_{x}\right\rangle_{(x, y, z)},\left\langle\phi_{y}, \phi_{y}\right\rangle=\left\langle\partial_{y}, \partial_{y}\right\rangle,\left\langle\phi_{z}, \phi_{z}\right\rangle=\left\langle\partial_{z}, \partial_{z}\right\rangle$, that is, $\phi$ is an isometry. Analogously, we can show that the rotation by angle $\pi$ around a horizontal geodesic parallel to the $x$-axis is also an isometry.

Remark 2.4. When the matrix $A$ in $\mathbb{R}^{2} \rtimes_{A} \mathbb{R}$ is $\left(\begin{array}{ll}0 & 1 \\ 0 & 0\end{array}\right)$ and $\left(\begin{array}{ll}0 & 1 \\ 1 & 0\end{array}\right)$, we have the Heisenberg space and $\mathrm{Sol}_{3}$, respectively, with their well-known Riemannian metrics. When we consider the one-parameter family of matrices $A(c)=\left(\begin{array}{cc}0 & c \\ 1 / c & 0\end{array}\right), c \geq 1$, we get a oneparameter family of metrics in $\mathrm{Sol}_{3}$ which are not isometric. For more details, see [4].

Meeks et al. [3] have proved results concerning the geometry of solutions to Plateau type problems in metric semidirect products $\mathbb{R}^{2} \rtimes_{A} \mathbb{R}$, when there is some geometric constraint on the boundary values of the solution. More precisely, they proved the following theorem.

TheOREM 2.5 (Meeks et al. [3]). Let $X=\mathbb{R}^{2} \rtimes_{A} \mathbb{R}$ be a metric semidirect product with its canonical metric and let $\Pi: \mathbb{R}^{2} \rtimes_{A} \mathbb{R} \rightarrow \mathbb{R}^{2} \rtimes_{A}\{0\}$ denote the projection $\Pi(x, y, z)=$ $(x, y, 0)$. Suppose that $E$ is a compact convex disk in $\mathbb{R}^{2} \rtimes_{A}\{0\}, C=\partial E$ and $\Gamma \subset \Pi^{-1}(C)$ is a continuous simple closed curve such that $\Pi: \Gamma \rightarrow C$ monotonically parameterizes C. Then:

(1) $\Gamma$ is the boundary of a compact embedded disk $\Sigma$ of finite least area;

(2) the interior of $\Sigma$ is a smooth $\Pi$-graph over the interior of $E$.

\section{A doubly periodic Scherk minimal surface}

Throughout this section, we consider the semidirect product $\mathbb{R}^{2} \rtimes_{A} \mathbb{R}$ with the canonical left invariant metric $\langle$,$\rangle , where A=\left(\begin{array}{ll}0 & b \\ c & 0\end{array}\right)$. In this space, we prove the 
existence of a complete minimal surface analogous to Scherk's doubly periodic minimal surface in $\mathbb{R}^{3}$.

Fix $0<c_{0}<c_{1}$ and let $a$ be a sufficiently small positive quantity such that

$$
\begin{aligned}
a< & \int_{c_{0}}^{c_{1}} \sqrt{a_{11}^{2}(z)+a_{21}^{2}(z)}+\sqrt{a_{11}^{2}(z)+a_{12}^{2}(z)} d z \\
& -\int_{c_{0}}^{c_{1}} \sqrt{\left(a_{11}(z)+a_{12}(z)\right)^{2}+\left(a_{11}(z)+a_{21}(z)\right)^{2}} d z .
\end{aligned}
$$

Note that such positive number $a$ exists, as

$$
\left|\partial_{x}\right|=\sqrt{a_{11}^{2}(z)+a_{21}^{2}(z)}, \quad\left|\partial_{y}\right|=\sqrt{a_{11}^{2}(z)+a_{12}^{2}(z)}
$$

and

$$
\left|\partial_{x}+\partial_{y}\right|=\sqrt{\left(a_{11}(z)+a_{12}(z)\right)^{2}+\left(a_{11}(z)+a_{21}(z)\right)^{2}} .
$$

For each $c>0$, consider the polygon $P_{c}$ in $\mathbb{R}^{2} \rtimes_{A} \mathbb{R}$ with sides $\alpha_{1}, \alpha_{2}, \alpha_{3}^{c}, \alpha_{4}^{c}$ and $\alpha_{5}^{c}$ defined by

$$
\begin{aligned}
\alpha_{1} & =\{(t, 0,0): 0 \leq t \leq a\}, \\
\alpha_{2} & =\{(0, t, 0): 0 \leq t \leq a\}, \\
\alpha_{3}^{c} & =\{(a, 0, t): 0 \leq t \leq c\}, \\
\alpha_{4}^{c} & =\{(0, a, t): 0 \leq t \leq c\}, \\
\alpha_{5}^{c} & =\{(t,-t+a, c): 0 \leq t \leq a\},
\end{aligned}
$$

as illustrated in Figure 1.

We will denote $\alpha_{1}^{0}=\{(t, 0,0): 0 \leq t<a\}, \alpha_{2}^{0}=\{(0, t, 0): 0 \leq t<a\}, \alpha_{3}=\{(a, 0, t)$ : $t>0\}$ and $\alpha_{4}=\{(0, a, t): t>0\}$, hence $P_{\infty}=\alpha_{1}^{0} \cup \alpha_{2}^{0} \cup \alpha_{3} \cup \alpha_{4} \cup\{(a, 0,0),(0, a, 0)\}$.

Let $\Pi: \mathbb{R}^{2} \rtimes_{A} \mathbb{R} \rightarrow \mathbb{R}^{2} \rtimes_{A}\{0\}$ denote the projection $\Pi(x, y, z)=(x, y, 0)$. The next proposition is proved in [3, Lemma 1.2], using the maximum principle and the fact that for every line $L \subset \mathbb{R}^{2} \rtimes_{A}\{0\}$, the vertical plane $\Pi^{-1}(L)$ is a minimal surface.

Proposition 3.1. Let $E$ be a compact convex disk in $\mathbb{R}^{2} \rtimes_{A}\{0\}$ with boundary $C=\partial E$ and let $\Sigma$ be a compact minimal surface with boundary in $\Pi^{-1}(C)$. Then every point in int $\Sigma$ is contained in int $\Pi^{-1}(E)$.

Observe that, for each $c>0$, the polygon $P_{c}$ is transverse to the Killing field $X=\partial_{x}+\partial_{y}$ and each integral curve of $X$ intersects $P_{c}$ at at most one point. From now on, denote by $P$ the common projection of every $P_{c}$ over $\mathbb{R}^{2} \rtimes_{A}\{0\}$, that is, $P=\Pi\left(P_{c}\right)=\Pi\left(P_{d}\right)$ for any $c, d \in \mathbb{R}$, and denote by $E$ the disk in $\mathbb{R}^{2} \rtimes_{A}\{0\}$ with boundary $P$. Let us denote by $\mathcal{R}$ the region $E \times\{z \geq 0\}$. Using Theorem 2.5, we conclude that $P_{c}$ is the boundary of a compact embedded disk $\Sigma_{c}$ of finite least area and the interior of $\Sigma_{c}$ is a smooth $\Pi$-graph over the interior of $E$.

Let $\Omega_{c}=\{(t,-t+a, s): 0 \leq t \leq a ; 0 \leq s \leq c\}$. 


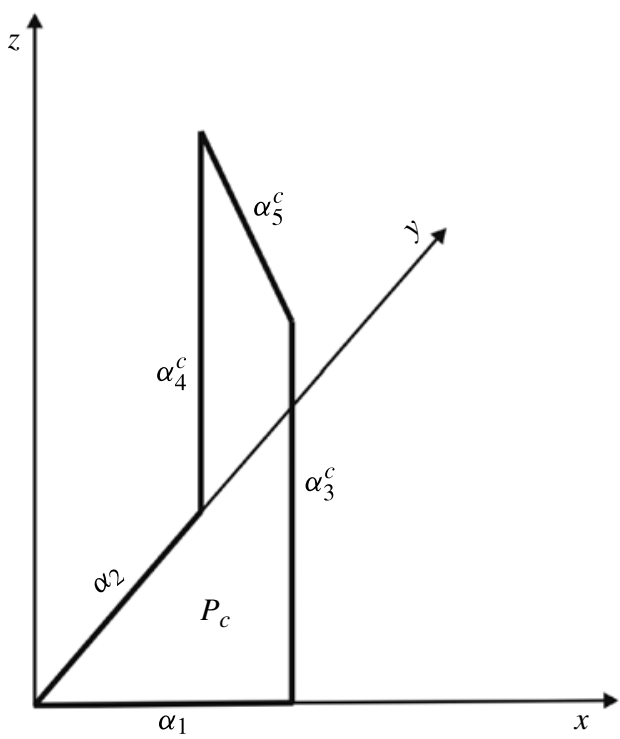

FIgURe 1. Polygon $P_{c}$.

Proposition 3.2. If $S$ is a compact minimal surface with boundary $P_{c}$, then $S=\Sigma_{c}$.

Proof. By Proposition 3.1, int $\Sigma_{c}$, int $S \subset \operatorname{int} \Pi^{-1}(E)$; then, in particular, int $\Sigma_{c}$, int $S \subset$ $\operatorname{int}\left\{\varphi_{t}(p): t \in \mathbb{R} ; p \in \Omega_{c}\right\}$, where $\varphi_{t}$ is the flow of the Killing field $X$.

As $S$ is compact, there exists $t$ such that $\varphi_{t}\left(\Sigma_{c}\right) \cap S=\emptyset$. If $S \neq \Sigma_{c}$, then there exists $t_{0}>0$ such that $\varphi_{t_{0}}\left(\Sigma_{c}\right) \cap S \neq \emptyset$ and, for $t>t_{0}, \varphi_{t}\left(\Sigma_{c}\right) \cap S=\emptyset$. Since for all $t \neq 0, \varphi_{t}\left(P_{c}\right) \cap S=\emptyset$, the point of intersection is an interior point and, by the maximum principle, $\varphi_{t_{0}}\left(\Sigma_{c}\right)=S$. But that is a contradiction, since $t_{0} \neq 0$. Therefore, $S=\Sigma_{c}$.

The next proposition is a classical result.

Proposition 3.3. Let $N^{3}$ be a homogeneous 3-manifold. Let $\Sigma_{n}$ be an oriented, properly embedded minimal surface in $N$. Suppose that there exist $c>0$ such that for all $n$, $\left|A_{\Sigma_{n}}\right| \leq c$, and a sequence of points $\left\{p_{n}\right\}$ in $\Sigma_{n}$ such that $p_{n} \rightarrow p \in N$. Then there exists a subsequence of $\Sigma_{n}$ that converges to a complete minimal surface $\Sigma$ with $p \in \Sigma$. Here $A_{\Sigma_{n}}$ denotes the second fundamental form of $\Sigma_{n}$.

For each $n \in \mathbb{N}$, let $\Sigma_{n}$ be the solution to the Plateau problem with boundary $P_{n}$. By Theorem 2.5 and Proposition 3.2, $\Sigma_{n}$ is stable and unique. We are interested in proving the existence of a subsequence of $\Sigma_{n}$ that converges to a complete minimal surface with boundary $P_{\infty}$. In order to do so, we will use a minimal annulus as a barrier (whose existence is guaranteed by the Douglas criterion (see [1, Theorem 2.1])) to show that there exist points $p_{n} \in \Sigma_{n}, \Pi\left(p_{n}\right)=q \in \operatorname{int} E$ for all $n$, which converge to a point $p \in \mathbb{R}^{2} \rtimes_{A} \mathbb{R}$, and then we will use Proposition 3.3. 
Consider the parallelepiped with the faces $A, B, C, D, E$ and $F$, defined by

$$
\begin{aligned}
& A=\left\{(u,-\epsilon, v): \epsilon \leq u \leq a+\epsilon ; c_{0} \leq v \leq c_{1}\right\}, \\
& B=\left\{(-\epsilon, u, v): \epsilon \leq u \leq a+\epsilon ; c_{0} \leq v \leq c_{1}\right\}, \\
& C=\left\{(u,-u, v):-\epsilon \leq u \leq \epsilon ; c_{0} \leq v \leq c_{1}\right\}, \\
& D=\left\{(u,-u+a, v):-\epsilon \leq u \leq a+\epsilon ; c_{0} \leq v \leq c_{1}\right\}, \\
& E=\left\{\left(u,-u+v, c_{0}\right):-\epsilon \leq u \leq v+\epsilon ; 0 \leq v \leq a\right\}, \\
& F=\left\{\left(u,-u+v, c_{1}\right):-\epsilon \leq u \leq v+\epsilon ; 0 \leq v \leq a\right\},
\end{aligned}
$$

where $\epsilon$ is a positive constant that we will choose later. Observe that each one of these faces is the least area minimal surface with its boundary. Let us analyse the area of each face.

(1) In the plane $\{y=$ constant $\}$ the induced metric is given by $g(x, z)=\left(a_{11}^{2}(z)+\right.$ $\left.a_{21}^{2}(z)\right) d x^{2}+d z^{2}$. Hence,

$$
\text { area } \begin{aligned}
A & =\int_{c_{0}}^{c_{1}} \int_{\epsilon}^{a+\epsilon} \sqrt{a_{11}^{2}(z)+a_{21}^{2}(z)} d x d z \\
& =a \int_{c_{0}}^{c_{1}} \sqrt{a_{11}^{2}(z)+a_{21}^{2}(z)} d z .
\end{aligned}
$$

(2) In the plane $\{x=$ constant $\}$ the induced metric is given by $g(y, z)=\left(a_{11}^{2}(z)+\right.$ $\left.a_{12}^{2}(z)\right) d y^{2}+d z^{2}$. Hence,

$$
\text { area } \begin{aligned}
B & =\int_{c_{0}}^{c_{1}} \int_{\epsilon}^{a+\epsilon} \sqrt{a_{11}^{2}(z)+a_{12}^{2}(z)} d x d z \\
& =a \int_{c_{0}}^{c_{1}} \sqrt{a_{11}^{2}(z)+a_{12}^{2}(z)} d z .
\end{aligned}
$$

(3) The face $C$ is contained in the plane parameterized by $\phi(u, v)=(u,-u, v)$ and the face $D$ is contained in the plane parameterized by $\psi(u, v)=(u,-u+a, v)$. We have $\psi_{u}=\phi_{u}=\partial_{x}-\partial_{y}, \psi_{v}=\phi_{v}=\partial_{z}$. Then

$$
\left|\psi_{u} \wedge \psi_{v}\right|=\left|\phi_{u} \wedge \phi_{v}\right|=\sqrt{\left(a_{11}(z)+a_{12}(z)\right)^{2}+\left(a_{11}(z)+a_{21}(z)\right)^{2}} .
$$

Hence,

$$
\begin{aligned}
\operatorname{area} C & =\int_{c_{0}}^{c_{1}} \int_{-\epsilon}^{+\epsilon} \sqrt{\left(a_{11}(z)+a_{12}(z)\right)^{2}+\left(a_{11}(z)+a_{21}(z)\right)^{2}} d u d v \\
& =2 \epsilon \int_{c_{0}}^{c_{1}} \sqrt{\left(a_{11}(z)+a_{12}(z)\right)^{2}+\left(a_{11}(z)+a_{21}(z)^{2}\right.} \mathrm{d} z, \\
\text { area } D & =\int_{c_{0}}^{c_{1}} \int_{-\epsilon}^{a+\epsilon} \sqrt{\left(a_{11}(z)+a_{12}(z)\right)^{2}+\left(a_{11}(z)+a_{21}(z)\right)^{2}} d u d v \\
& =(a+2 \epsilon) \int_{c_{0}}^{c_{1}} \sqrt{\left(a_{11}(z)+a_{12}(z)\right)^{2}+\left(a_{11}(z)+a_{21}(z)\right)^{2}} d z .
\end{aligned}
$$


(4) As the plane $\{z=$ constant $\}$ is flat, the induced metric is the Euclidean metric. Hence,

$$
\text { area } E=\text { area } F=\int_{0}^{a} \int_{-\epsilon}^{v+\epsilon} d u d v=\frac{a(a+4 \epsilon)}{2} .
$$

Therefore,

$$
\text { area } C+\text { area } D+\text { area } E+\text { area } F<\text { area } A+\text { area } B
$$

if, and only if,

$$
\begin{aligned}
(a+4 \epsilon)\left[a+\int_{c_{0}}^{c_{1}} \sqrt{\left(a_{11}+a_{12}\right)^{2}+\left(a_{11}+a_{21}\right)^{2}} d z\right]<a & \int_{c_{0}}^{c_{1}} \sqrt{a_{11}^{2}+a_{21}^{2}} d z \\
& +a \int_{c_{0}}^{c_{1}} \sqrt{a_{11}^{2}+a_{12}^{2}} d z
\end{aligned}
$$

if, and only if,

$$
\epsilon<\frac{a}{4} \frac{\int_{c_{0}}^{c_{1}} \sqrt{a_{11}^{2}(z)+a_{21}^{2}(z)}+\sqrt{a_{11}^{2}(z)+a_{21}^{2}(z)} d z}{a+\int_{c_{0}}^{c_{1}} \sqrt{\left(a_{11}(z)+a_{12}(z)\right)^{2}+\left(a_{11}(z)+a_{21}(z)\right)^{2}} d z}-\frac{a}{4} .
$$

As we chose $a$ satisfying (3.1), the factor on the right-hand side of (3.2) is a positive number, and we can choose $\epsilon>0$ such that the Douglas criterion is satisfied [1]. Hence we obtain a minimal annulus $\mathcal{A}$ with boundary $\partial A \cup \partial B$ such that its projection $\Pi(\mathcal{A})$ contains points of int $E$, where $E$ is the disk in $\mathbb{R} \rtimes_{A}\{0\}$ with boundary $P$ (see Figure 2).

As $\mathbb{R}^{2} \rtimes_{A}\{z\}$ is a minimal surface, the maximum principle implies that, for each $c$, $\Sigma_{c}$ is contained in the slab bounded by the planes $\{z=0\}$ and $\{z=c\}$. Then for $c<c_{0}$, $\Sigma_{c} \cap \mathcal{A}=\emptyset$. As $\Sigma_{c}$ is unique, $\Sigma_{c}$ varies continuously with $c$, and when $c$ increases the boundary $\partial \Sigma_{c}=P_{c}$ does not touch $\partial \mathcal{A}$. Therefore, using the maximum principle, $\Sigma_{c} \cap \mathcal{A}=\emptyset$ for all $c$, and $\Sigma_{c}$ is under the annulus $\mathcal{A}$, which means that over any vertical line that intersects $\mathcal{A}$ and $\Sigma_{c}$, the points of $\Sigma_{c}$ are under the points of $\mathcal{A}$.

Consider the flow $\varphi_{t}$ of the Killing field $X=\partial_{x}+\partial_{y}$. Observe that $\left\{\varphi_{t}(\mathcal{A})\right\}_{t<0}$ forms a barrier for all points $p_{n} \in \Sigma_{n}$ such that $\Pi\left(p_{n}\right)$ is contained in a neighborhood $\mathcal{U} \subset E$ of the origin $o=(0,0,0)$. Moreover, for any $c_{2}<c_{3}$ we can use the flow $\varphi_{t}$ of the Killing field $X$ and the maximum principle to conclude that $\Sigma_{c_{2}}$ is under $\Sigma_{c_{3}}$ in the same sense as before.

As, by Theorem 2.5, each $\Sigma_{n}$ is a vertical graph in the interior, then $\Sigma_{n} \cap \Pi^{-1}(q)$ is only one point $p_{n}$, for every point $q \in \operatorname{int} E$. Moreover, by the previous paragraph, the sequence $p_{n}=\Sigma_{n} \cap \Pi^{-1}(q)$ is monotone. Then, since we have a barrier, the sequence $\left\{p_{n}=\Sigma_{n} \cap \Pi^{-1}(q)\right\}$ converges to a point $p \in \Pi^{-1}(q)$, for all $q \in \mathcal{U}$.

In order to understand the convergence of the surfaces $\Sigma_{n}$ we need to observe some properties of these surfaces.

First, notice that rotation by angle $\pi$ around $\alpha_{3}$, which we will denote by $R_{\alpha_{3}}$, is an isometry. By the Schwarz reflection, we obtain a minimal surface $\widetilde{\Sigma}_{n}=\Sigma_{n} \cup R_{\alpha_{3}}\left(\Sigma_{n}\right)$ that has int $\alpha_{3}$ in its interior. Note that the boundary of $\widetilde{\Sigma}_{n}$ is transverse to the Killing 


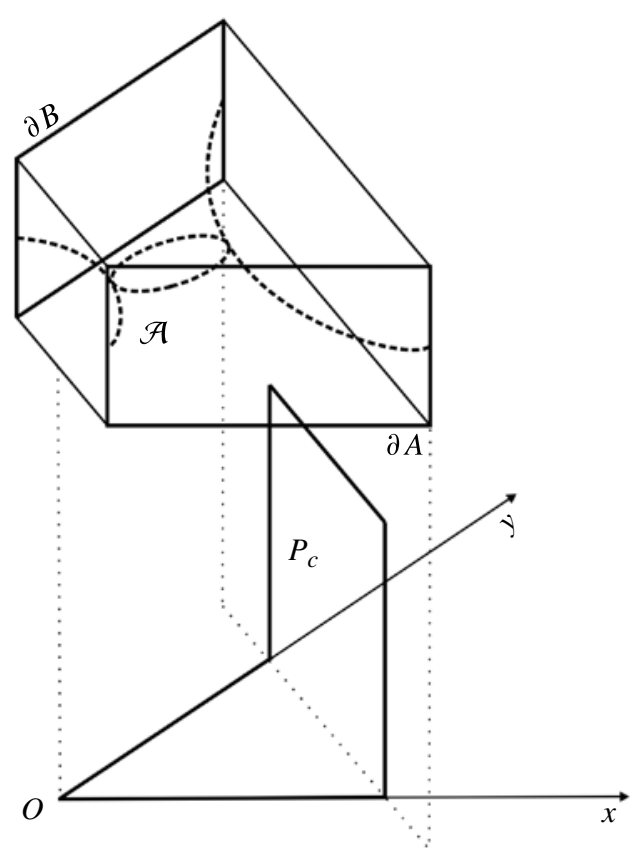

Figure 2. Annulus $\mathcal{A}$.

field $X=\partial_{x}+\partial_{y}$, and if $\varphi_{t}$ denotes the flow of $X$, we have that $\varphi_{t}\left(\partial \widetilde{\Sigma}_{n}\right) \cap \widetilde{\Sigma}_{n}=\emptyset$ for all $t \neq 0$, hence, using the same arguments of the proof of Proposition 3.2, we can show that the minimal surface $\widetilde{\Sigma}_{n}$ is the unique minimal surface with its boundary. In particular, it is area-minimizing, and then it is stable. Hence, by main theorem in [6], we have uniform curvature estimates for points far from the boundary of $\widetilde{\Sigma}_{n}$. In particular, we get uniform curvature estimates for $\Sigma_{n}$ in a neighborhood of $\alpha_{3}$. Analogously, we have uniform curvature estimates for $\Sigma_{n}$ in a neighborhood of $\alpha_{4}$.

Hence, for every compact contained in $\{z>0\} \cap \mathcal{R}$, there exists a subsequence of $\Sigma_{n}$ that converges to a minimal surface. Taking exhaustion by compact sets and using a diagonal process, we conclude that there exists a subsequence of $\Sigma_{n}$ that converges to a minimal surface $\Sigma$ that has $\alpha_{3} \cup \alpha_{4}$ in its boundary. From now on, we will use the notation $\Sigma_{n}$ for this subsequence.

It remains to prove that in fact $\Sigma$ is a minimal surface with boundary $P_{\infty}$. In order to do so, we will use the fact that the interior of each $\Sigma_{n}$ is a vertical graph over the interior of $E$. Let us denote by $u_{n}$ the function defined in $\operatorname{int} E \operatorname{such}$ that $\Sigma_{n}=\operatorname{Graph}\left(u_{n}\right)$. We already know that $u_{n-1}<u_{n}$ in int $E$ for all $n$.

ClaIm 3.4. There are uniform gradient estimates for $\left\{u_{n}\right\}$ for points in $\alpha_{1}^{0} \cup \alpha_{2}^{0}$.

Proof. For $x_{0}<0$ and $\delta>0$ consider the vertical strip bounded by $\beta_{1}=\left\{\left(x_{0}, y, c_{1}\right)\right.$ : $-\delta \leq y \leq 0\}, \quad \beta_{2}=\left\{\left(x_{0}, t,-\left(c_{1} / a\right) t+c_{1}\right): 0 \leq t \leq a\right\}, \quad \beta_{3}=\left\{\left(x_{0}, t-\delta,-\left(c_{1} / a\right) t+c_{1}\right):\right.$ $0 \leq t \leq a\}$ and $\beta_{4}=\left\{\left(x_{0}, y, 0\right): a-\delta \leq y \leq a\right\}$. This is a minimal surface transversal 


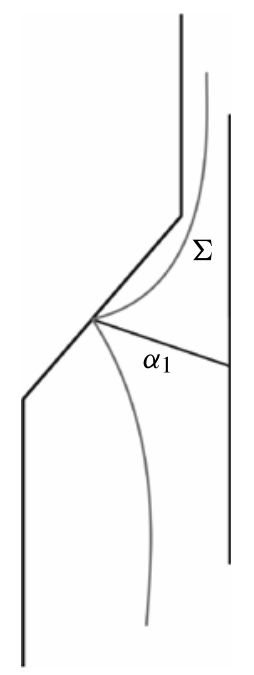

FigURE 3. Rotation by angle $\pi$ around $\alpha_{1}$ of $\Sigma$.

to the Killing field $\partial_{x}$, hence any small perturbation of its boundary gives a minimal surface with that perturbed boundary. Thus, if we consider a small perturbation of the boundary of this vertical strip by just slightly perturbing $\beta_{1}$ by a curve contained in $\left\{x \geq x_{0}\right\}$ joining the points $\left(x_{0},-\delta, c_{1}\right)$ and $\left(x_{0}, 0, c_{1}\right)$, we will get a minimal surface $S$ with this perturbed boundary. This minimal surface $S$ will have the property that the tangent planes at the interior of $\beta_{4}$ are not vertical, by the maximum principle with boundary.

Applying translations along the $x$-axis and $y$-axis, we can use the translates of $S$ to show that $\Sigma_{n}$ is under $S$ in a neighborhood of $\alpha_{2}^{0}$, and then we have uniform gradient estimates for points in $\alpha_{2}^{0}$. Analogously, constructing similar barriers, we can prove that we have uniform gradient estimates in a neighborhood of $\alpha_{1}^{0}$.

Observe that besides the gradient estimates, the translates of the minimal surface $S$ form a barrier for points in a neighborhood of $\alpha_{1}^{0} \cup \alpha_{2}^{0}$.

We have that $\Sigma_{n}$ is a monotone increasing sequence of minimal graphs with uniform gradient estimates in $\alpha_{1}^{0} \cup \alpha_{2}^{0}$, and it is a bounded graph for points in a neighborhood $\mathcal{U}$ of the origin (because of the barrier given by the annulus $\mathcal{A}$ ). Therefore, there exists a subsequence of $\Sigma_{n}$ that converges to a minimal surface $\widetilde{\Sigma}$ with $\alpha_{1}^{0} \cup \alpha_{2}^{0}$ in its boundary. As we already know that $\Sigma_{n}$ converges to the minimal surface $\Sigma$, we conclude that in fact $\Sigma=\widetilde{\Sigma}$, and then $\Sigma$ is a minimal surface with $\alpha_{1}^{0} \cup \alpha_{2}^{0} \cup \alpha_{3} \cup \alpha_{4}$ in its boundary. Notice that we can assume that $\Sigma$ has $P_{\infty}$ as its boundary, with $\Sigma$ being of class $C^{1}$ up to $P_{\infty} \backslash\{(a, 0,0),(0, a, 0)\}$ and continuous up to $P_{\infty}$.

Now considering the rotation by angle $\pi$ around $\alpha_{1}$ of $\Sigma$, we obtain the surface illustrated in Figure 3. 
Continuing to rotate by angle $\pi$ around the $y$-axis, the resulting surface will be a minimal surface with four vertical lines as its boundary: $\{(a, 0, t): t \in \mathbb{R}\},\{(0, a, t): t \in$ $\mathbb{R}\},\{(-a, 0, t): t \in \mathbb{R}\},\{(0,-a, t): t \in \mathbb{R}\}$.

Now we can use the rotations by angle $\pi$ around the vertical lines to get a complete minimal surface that is analogous to the doubly periodic minimal Scherk surface in $\mathbb{R}^{3}$. It is invariant with respect to two translations that commute and it is a four-punctured sphere in the quotient of $\mathbb{R}^{2} \rtimes_{A} \mathbb{R}$ by the group of isometries generated by the two translations.

THEOREM 3.5. In any semidirect product $\mathbb{R}^{2} \rtimes_{A} \mathbb{R}$, where $A=\left(\begin{array}{ll}0 & b \\ c & 0\end{array}\right)$, there exists a periodic minimal surface similar to the doubly periodic Scherk minimal surface in $\mathbb{R}^{3}$.

\section{A singly periodic Scherk minimal surface}

Throughout this section, we consider the semidirect product $\mathbb{R}^{2} \rtimes_{A} \mathbb{R}$ with the canonical left invariant metric $\langle$,$\rangle , where A=\left(\begin{array}{ll}0 & b \\ c & 0\end{array}\right)$. In this space, we construct a complete minimal surface similar to the singly periodic Scherk minimal surface in $\mathbb{R}^{3}$.

Fix $c_{0}>0$ and take $0<\epsilon<a$ sufficiently small so that

$$
a+2 \epsilon<\int_{0}^{c_{0}} \sqrt{a_{11}^{2}(z)+a_{21}^{2}(z)} d z
$$

For each $c>0$, consider the polygon $P_{c}$ in $\mathbb{R}^{2} \rtimes_{A} \mathbb{R}$ with the six sides defined by

$$
\begin{aligned}
& \alpha_{1}^{c}=\{(t, 0,0): 0 \leq t \leq c\}, \\
& \alpha_{2}^{c}=\{(c, t, 0): 0 \leq t \leq a\}, \\
& \alpha_{3}^{c}=\{(t, a, 0): 0 \leq t \leq c\}, \\
& \alpha_{4}^{c}=\{(0, a, t): 0 \leq t \leq c\}, \\
& \alpha_{5}^{c}=\{(0, t, c): 0 \leq t \leq a\}, \\
& \alpha_{6}^{c}=\{(0,0, t): 0 \leq t \leq c\},
\end{aligned}
$$

and for each $\delta>0$ with $\delta<a / 2$, consider the polygon $P_{c}^{\delta}$ with the six sides

$$
\begin{aligned}
& \alpha_{1}^{\delta, c}=\left\{\left(t, \frac{\delta}{c} t, 0\right): 0 \leq t \leq c\right\} \\
& \alpha_{2}^{\delta, c}=\{(c, t, 0): \delta \leq t \leq a-\delta\} \\
& \alpha_{3}^{\delta, c}=\left\{\left(t, \frac{a c-\delta t}{c}, 0\right): 0 \leq t \leq c\right\},
\end{aligned}
$$

$\alpha_{4}^{c}, \alpha_{5}^{c}, \alpha_{6}^{c}$, as illustrated in Figure 4 .

Denote by $\Omega(\delta, c)$ the region in $\mathbb{R}^{2} \rtimes_{A}\{0\}$ bounded by $\alpha_{1}^{\delta, c}, \alpha_{2}^{\delta, c}, \alpha_{3}^{\delta, c}$ and the segment $\{(0, t, 0): 0 \leq t \leq a\}$. For each $c$ and $\delta$, we have compact minimal surfaces $\Sigma_{c}$ and $\Sigma_{c}^{\delta}$ with boundary $P_{c}$ and $P_{c}^{\delta}$, respectively, which are solutions to the Plateau problem. By Theorem 2.5, we know that $\Sigma_{c}$ and $\Sigma_{c}^{\delta}$ are stable and smooth $\Pi$-graphs over the interior 

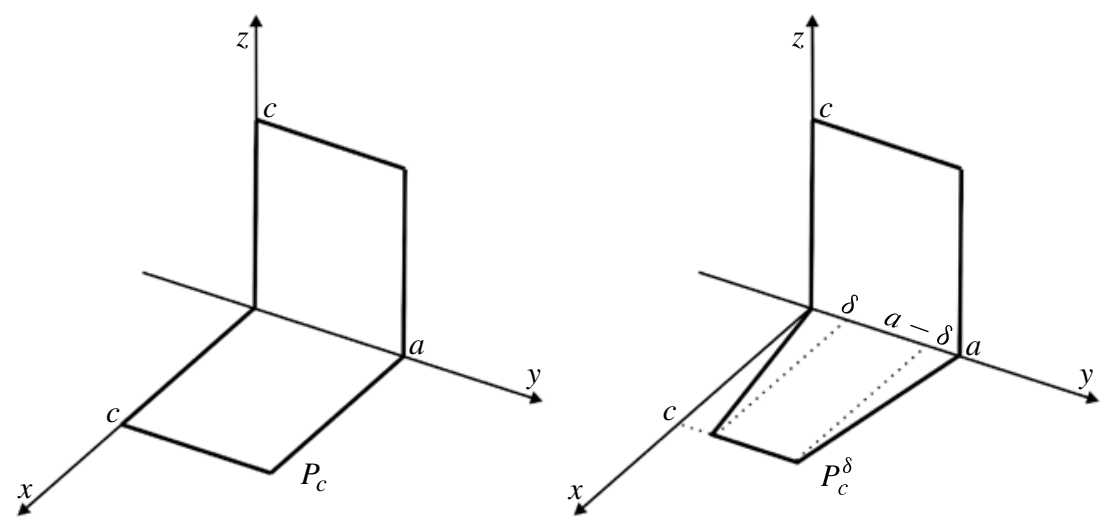

Figure 4. Polygons $P_{c}$ and $P_{c}^{\delta}$.

of $\Omega(0, c), \Omega(\delta, c)$, respectively. We will show that $\Sigma_{c}$ is the unique compact minimal surface with boundary $P_{c}$.

Fix $c$. For each $0<\delta<a / 2, P_{c}^{\delta}$ is a polygon transverse to the Killing field $\partial_{x}$ and each integral curve of $\partial_{x}$ intersects $P_{c}^{\delta}$ at at most one point. Thus we can prove, as we did in Proposition 3.2, that $\Sigma_{c}^{\delta}$ is the unique compact minimal surface with boundary $P_{c}^{\delta}$.

Denote by $u_{c}^{\delta}, v_{c}$ the functions defined in the interior of $\Omega(\delta, c), \Omega(0, c)$, whose $\Pi$ graphs are $\Sigma_{c}^{\delta}, \Sigma_{c}$, respectively. Then, as $\partial_{x}$ is a Killing field and each $P_{c}^{\delta}$ is transversal to $\partial_{x}$, we can use the flow of $\partial_{x}$ and the maximum principle to prove that for $\delta^{\prime}<\delta$ we have $0 \leq u_{c}^{\delta} \leq u_{c}^{\delta^{\prime}} \leq v_{c}$ in int $\Omega(\delta, c)$, hence $v_{c}$ is a barrier for our sequence $u_{c}^{\delta}$. Because of the monotonicity and the barrier, the family $u_{c}^{\delta}$ converges to a function $u_{c}$ defined in int $\Omega(0, c)$ whose graph is a compact minimal surface with boundary $P_{c}$, and we still have $u_{c} \leq v_{c}$ on $\Omega(0, c)$.

We will now find another compact minimal surface with boundary $P_{c}$, whose interior is the graph of a function $w_{c}$ defined in int $\Omega(0, c)$ such that $v_{c} \leq w_{c}$ and we will show that $u_{c}=w_{c}$. In order to do so, for each $0<\delta<a / 2$, consider the polygon $\widetilde{P}_{c}^{\delta}$ with the six sides defined by

$$
\begin{aligned}
\widetilde{\alpha}_{1}^{\delta, c} & =\left\{\left(t, \frac{\delta t-\delta c}{c}, 0\right): 0 \leq t \leq c\right\}, \\
\alpha_{2}^{c} & =\{(c, t, 0): 0 \leq t \leq a\}, \\
\widetilde{\alpha}_{3}^{\delta, c} & =\left\{\left(t, \frac{(a+\delta) c-\delta t}{c}, 0\right): 0 \leq t \leq c\right\}, \\
\widetilde{\alpha}_{4}^{\delta, c} & =\{(0, a+\delta, t): 0 \leq t \leq c\}, \\
\widetilde{\alpha}_{5}^{\delta, c} & =\{(0, t, c):-\delta \leq t \leq a+\delta\}, \\
\widetilde{\alpha}_{6}^{\delta, c} & =\{(0,-\delta, t): 0 \leq t \leq c\},
\end{aligned}
$$

as illustrated in Figure 5. 

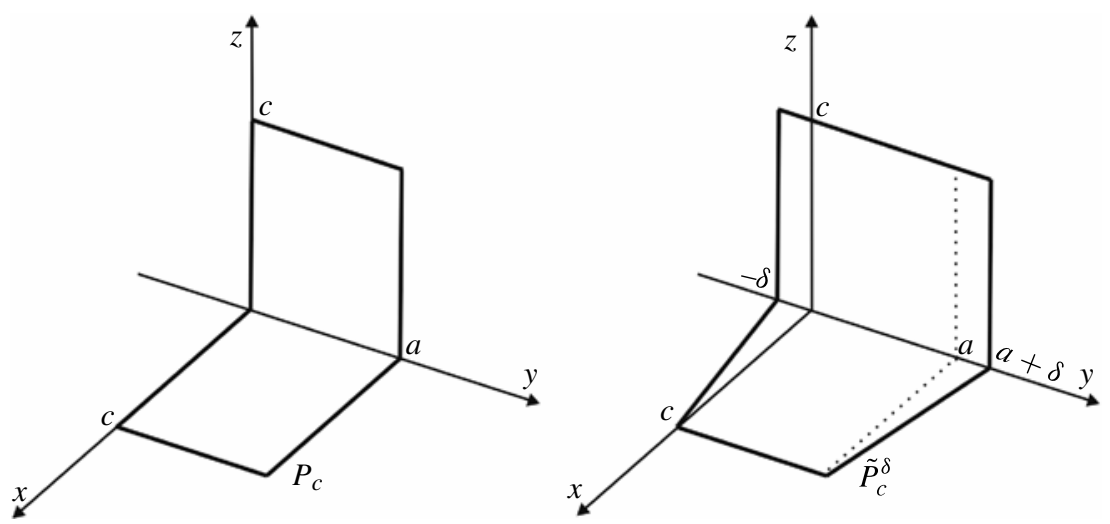

FIGURE 5. Polygons $P_{c}$ and $\widetilde{P}_{c}^{\delta}$.

Denote by $\widetilde{\Omega}(\delta, c)$ the region in $\mathbb{R}^{2} \rtimes_{A}\{0\}$ bounded by $\widetilde{\alpha}_{1}^{\delta, c}, \alpha_{2}^{c}, \widetilde{\alpha}_{3}^{\delta, c}$ and the segment $\{(0, t, 0):-\delta \leq t \leq a+\delta\}$. For each $\delta$, we have a compact minimal disk $\widetilde{\Sigma}_{c}^{\delta}$ with boundary $\widetilde{P}_{c}^{\delta}$ and $\widetilde{\Sigma}_{c}^{\delta}$ is a smooth $\Pi$-graph over the interior of $\widetilde{\Omega}(\delta, c)$. As $\widetilde{P}_{c}^{\delta}$ is transversal to the Killing field $\partial_{x}$, we can prove that $\widetilde{\Sigma}_{c}^{\delta}$ is the unique compact minimal surface with boundary $\widetilde{P}_{c}^{\delta}$.

Denote by $w_{c}^{\delta}$ the function defined in int $\widetilde{\Omega}(\delta, c)$ whose graph is $\widetilde{\Sigma}_{c}^{\delta}$. Using the flow of $\partial_{x}$ and the maximum principle, we can prove that for $\delta^{\prime}<\delta$ we have $w_{c}^{\delta^{\prime}} \leq w_{c}^{\delta}$ in $\operatorname{int} \widetilde{\Omega}\left(\delta^{\prime}, c\right)$ and for all $\delta, v_{c} \leq w_{c}^{\delta}$ in int $\Omega(0, c)$. Because of the monotonicity and the barrier, the family $w_{c}^{\delta}$ converges to a function $w_{c}$ defined in $\operatorname{int} \widetilde{\Omega}(0, c)=\operatorname{int} \Omega(0, c)$ whose graph is a compact minimal surface with boundary $P_{c}$, and we still have $v_{c} \leq w_{c}$ in int $\Omega(0, c)$.

Let us call $\Sigma_{1}, \Sigma_{2}$ the graphs of $u_{c}, w_{c}$, respectively. We will now prove that $\Sigma_{1}=\Sigma_{2}$. Denote by $v_{i}$ the conormal to $\Sigma_{i}$ along $P_{c}, i=1,2$. (See Figure 6.)

Suppose that $u_{c} \neq w_{c}$; then in fact we have $u_{c}<w_{c}$ in int $\Omega(0, c)$. As $\partial_{x}$ is tangent to $\alpha_{1}^{c}$ and $\alpha_{3}^{c}$, we have that $\left\langle v_{i}, \partial_{x}\right\rangle=0, i=1,2$, in $\alpha_{1}^{c}$ and $\alpha_{3}^{c}$. On the other sides of $P_{c}$ we have $\left\langle v_{1}, \partial_{x}\right\rangle<\left\langle v_{2}, \partial_{x}\right\rangle$. Therefore,

$$
\int_{P_{c}}\left\langle v_{1}, \partial_{x}\right\rangle<\int_{P_{c}}\left\langle v_{2}, \partial_{x}\right\rangle .
$$

But, using the flux formula for $\Sigma_{1}$ and $\Sigma_{2}$ with respect to the Killing field $\partial_{x}$,

$$
\int_{P_{c}}\left\langle v_{1}, \partial_{x}\right\rangle=0=\int_{P_{c}}\left\langle v_{2}, \partial_{x}\right\rangle .
$$

Then $u_{c}=w_{c}$, and therefore $\Sigma_{c}=\Sigma_{1}=\Sigma_{2}$. In particular, $\Sigma_{c}$ is the unique compact minimal surface with boundary $P_{c}$. 


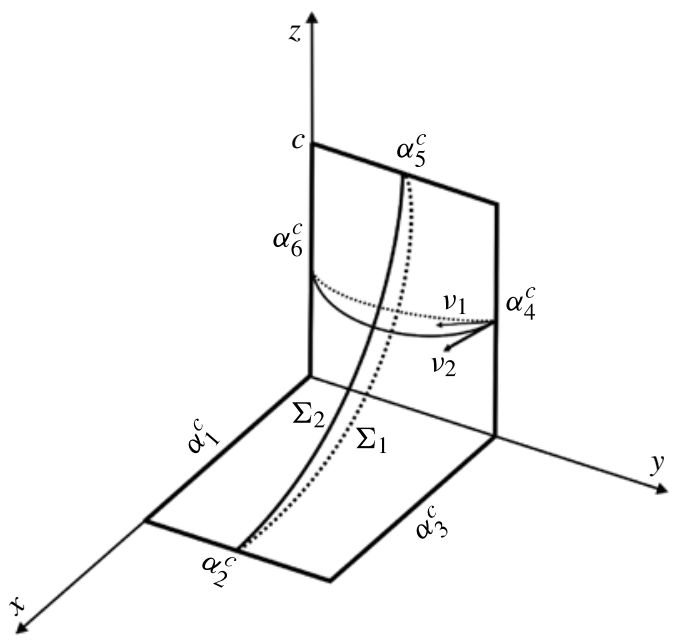

FIGURE 6. $\Sigma_{1}$ and $\Sigma_{2}$.

Denote by $\Omega(\infty)$ the infinite strip $\{(x, y, 0): x \geq 0,0 \leq y \leq a\}$, and by $\mathcal{R}$ the region $\{(x, y, z): x \geq 0,0 \leq y \leq a, z \geq 0\}$. Moreover, denote $\alpha_{1}=\{(x, 0,0): x>0\}$, $\alpha_{3}=\{(x, a, 0): x>0\}, \alpha_{4}=\{(0, a, z): z>0\}$ and $\alpha_{6}=\{(0,0, z): z>0\}$, hence $P_{\infty}=$ $\alpha_{1} \cup \alpha_{3} \cup \alpha_{4} \cup \alpha_{6} \cup\{(0,0,0),(0, a, 0)\}$.

For each $n \in \mathbb{N}$, let $\Sigma_{n}$ be the unique compact minimal surface with boundary $P_{n}$. We are interested in proving the existence of a subsequence of $\Sigma_{n}$ that converges to a complete minimal surface with boundary $P_{\infty}$. Using the existence of a minimal annulus, guaranteed by the Douglas criterion, we will show that there exist points $p_{n} \in \Sigma_{n}, \Pi\left(p_{n}\right)=q \in$ int $\Omega(\infty)$ for all $n$, which converge to a point $p \in \mathbb{R}^{2} \rtimes_{A} \mathbb{R}$, and then we will use Proposition 3.3.

Consider the parallelepiped with faces $A, B, C, D, E$ and $F$, defined by

$$
\begin{aligned}
& A=\left\{(u,-\epsilon, v): \epsilon \leq u \leq d ; 0 \leq v \leq c_{0}\right\}, \\
& B=\left\{(u, a+\epsilon, v): \epsilon \leq u \leq d ; 0 \leq v \leq c_{0}\right\}, \\
& C=\{(u, v, 0): \epsilon \leq u \leq d ;-\epsilon \leq v \leq a+\epsilon\}, \\
& D=\left\{\left(u, v, c_{0}\right): \epsilon \leq u \leq d ;-\epsilon \leq v \leq a+\epsilon\right\}, \\
& E=\left\{(\epsilon, u, v):-\epsilon \leq u \leq a+\epsilon ; 0 \leq v \leq c_{0}\right\}, \\
& F=\left\{(d, u, v):-\epsilon \leq u \leq a+\epsilon ; 0 \leq v \leq c_{0}\right\},
\end{aligned}
$$

where $d>\epsilon$ is a constant that we will choose later. 
As we did in the previous section, we can calculate the area of each one of these faces and we obtain:

$$
\begin{aligned}
& \text { area } A=\operatorname{area} B=(d-\epsilon) \int_{0}^{c_{0}} \sqrt{a_{11}^{2}(z)+a_{21}^{2}(z)} d z, \\
& \text { area } C=\operatorname{area} D=(d-\epsilon)(a+2 \epsilon), \\
& \text { area } E=\operatorname{area} F=(a+2 \epsilon) \int_{0}^{c_{0}} \sqrt{a_{11}^{2}(z)+a_{12}^{2}(z)} d z .
\end{aligned}
$$

Hence,

$$
\text { area } C+\text { area } D+\text { area } E+\text { area } F<\text { area } A+\text { area } B
$$

if, and only if,

$$
(d-\epsilon)(a+2 \epsilon)+(a+2 \epsilon) \int_{0}^{c_{0}} \sqrt{a_{11}^{2}+a_{12}^{2}} d z<(d-\epsilon) \int_{0}^{c_{0}} \sqrt{a_{11}^{2}+a_{21}^{2}} d z
$$

if, and only if,

$$
(d-\epsilon)\left[(a+2 \epsilon)-\int_{0}^{c_{0}} \sqrt{a_{11}^{2}+a_{21}^{2}} d z\right]<-(a+2 \epsilon) \int_{0}^{c_{0}} \sqrt{a_{11}^{2}+a_{12}^{2}} d z
$$

if, and only if,

$$
d>\epsilon-\frac{(a+2 \epsilon) \int_{0}^{c_{0}} \sqrt{a_{11}^{2}(z)+a_{12}^{2}(z)} d z}{(a+2 \epsilon)-\int_{0}^{c_{0}} \sqrt{a_{11}^{2}(z)+a_{21}^{2}(z)} d z} .
$$

As we chose $a+2 \epsilon<\int_{0}^{c_{0}} \sqrt{a_{11}^{2}(z)+a_{21}^{2}(z)} d z$, we can choose $d>\epsilon$ so that the Douglas criterion is satisfied [1]. Thus, there exists a minimal annulus $\mathcal{A}$ with boundary $\partial A \cup \partial B$ such that its projection $\Pi(\mathcal{A})$ contains points of int $\Omega(\infty)$. (See Figure 7.)

We know that, for each $c<\epsilon, \Sigma_{c} \cap \mathcal{A}=\emptyset$. When $c$ increases $P_{c}$ does not intersect $\partial \mathcal{A}$; then, using the maximum principle, $\Sigma_{c} \cap \mathcal{A}=\emptyset$ for all $c$, and $\Sigma_{c}$ is under the annulus $\mathcal{A}$. Thus, there exists a point $q \in \operatorname{int} \Omega(\infty)$ such that $p_{n}=\Sigma_{n} \cap \Pi^{-1}(q)$ has a subsequence that converges to a point $p \in \Pi^{-1}(q)$. Observe that, applying the flow of the Killing field $\partial_{x}$ to the annulus $\mathcal{A}$, we can conclude that, in the region $\{x \geq d\}$, the surfaces $\Sigma_{n}$ are bounded above by, for example, the plane $\left\{z=c_{0}\right\}$.

In order to understand the convergence of the surfaces $\Sigma_{n}$ we need to prove some properties of these surfaces.

Claim 4.1. The surfaces $\Sigma_{n}$ are transversal to the Killing field $\partial_{x}$ in the interior.

Proof. Fix $n$. Suppose that at some point $p \in \operatorname{int} \Sigma_{n}$ the tangent plane $T_{p} \Sigma_{n}$ contains the vector $\partial_{x}$. As the planes that contain the direction $\partial_{x}$ are minimal surfaces, we 


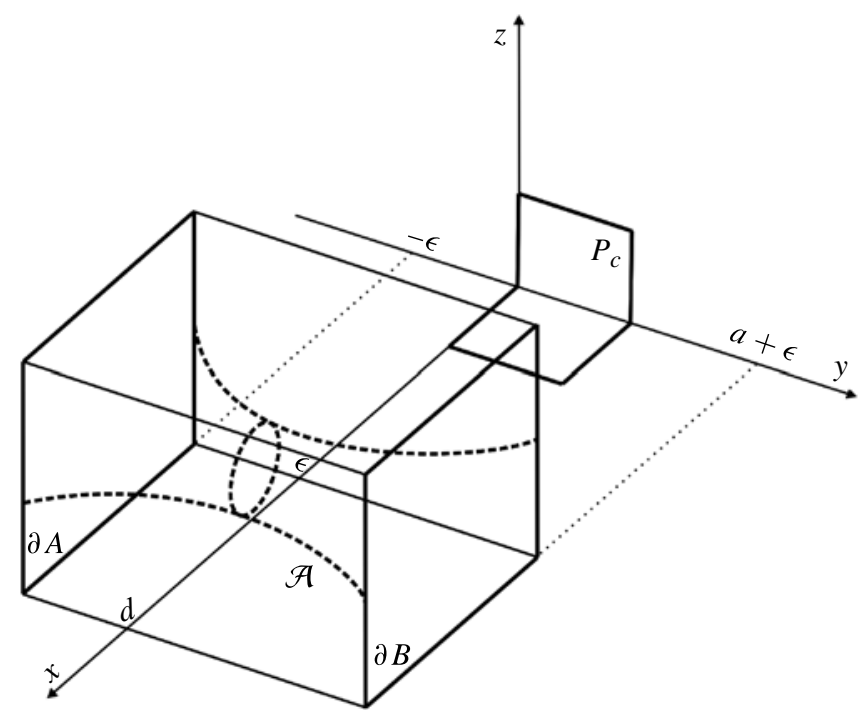

Figure 7. Annulus $\mathcal{A}$.

have that $\Sigma_{n}$ and $T_{p} \Sigma_{n}$ are minimal surfaces tangent at $p$, and then the intersection between them is formed by $2 k$ curves, $k \geq 1$, passing through $p$ making equal angles at $p$. By the shape of $P_{n}$ (the boundary of $\Sigma_{n}$ ), we know that $T_{p} \Sigma_{n}$ intersects $P_{n}$ either at only two points or at one point and a segment of straight line $\left(\alpha_{1}^{n}\right.$ or $\left.\alpha_{3}^{n}\right)$. Therefore, we will necessarily have a closed curve contained in the intersection. As $\Sigma_{n}$ is simply connected this curve bounds a disk in $\Sigma_{n}$, but as the planes parallel to $T_{p} \Sigma_{n}$ are minimal surfaces, we can use the maximum principle to prove that this disk is contained in the plane $T_{p} \Sigma_{n}$ and then they coincide, which is impossible. Thus, the vector $\partial_{x}$ is transversal to $\Sigma$ at points $p \in \operatorname{int} \Sigma_{n}$.

Observe that, besides the interior points, the surfaces $\Sigma_{n}$ are also transversal to $\partial_{x}$ at the points in $\alpha_{4}$ and $\alpha_{6}$, by the maximum principle with boundary. Thus rotation by angle $\pi$ around $\alpha_{4}$ (respectively $\alpha_{6}$ ) gives a minimal surface which is also transversal to the Killing field $\partial_{x}$ in the interior, extends the surface $\Sigma_{n}$ and has $\alpha_{4}^{n}$ (respectively $\alpha_{6}^{n}$ ) in the interior. Therefore, we have uniform curvature estimates for $\Sigma_{n}$ up to $\alpha_{4} \cup \alpha_{6}$.

Hence, for every compact contained in $\{z>0\} \cap \mathcal{R}$, there exists a subsequence of $\Sigma_{n}$ that converges to a minimal surface. Taking exhaustion by compact sets and using a diagonal process, we conclude that there exists a subsequence of $\Sigma_{n}$ that converges to a minimal surface $\Sigma$ that has $\alpha_{4} \cup \alpha_{6}$ in its boundary. From now on we will use the notation $\Sigma_{n}$ for this subsequence.

It remains to prove that in fact $\Sigma$ is a minimal surface with boundary $P_{\infty}$. In order to do so, we will use the fact that each $\Sigma_{n}$ is a vertical graph in the interior. 
Let us denote by $u_{n}$ the function defined in $\operatorname{int} \Omega(n)$ such that $\Sigma_{n}=\operatorname{Graph}\left(u_{n}\right)$, where $\Omega(n)=\{(x, y, 0): 0 \leq x \leq n ; 0 \leq y \leq a\}$.

ClaIm 4.2. $u_{n-1}<u_{n}$ in $\operatorname{int} \Omega(n-1)$.

Proof. Recall that each $\Sigma_{n}$ is the limit of a sequence of minimal graphs $\widetilde{\Sigma}_{n}^{\delta}=\operatorname{Graph}\left(w_{n}^{\delta}\right)$ whose boundary is transversal to the Killing field $\partial_{x}$. Using the flow of the Killing field $\partial_{x}$, we can prove that each $\widetilde{\Sigma}_{n}^{\delta}$ is above $\Sigma_{n-1}$, and then the limit surface $\Sigma_{n}$ has to be above $\Sigma_{n-1}$. In fact, $\Sigma_{n}$ is strictly above $\Sigma_{n-1}$ in the interior, because as $\Sigma_{n}$ and $\Sigma_{n-1}$ are minimal surfaces, if they intersect at an interior point, there will be points of $\Sigma_{n}$ under $\Sigma_{n-1}$, and we already know that, by the property of $\widetilde{\Sigma}_{n}^{\delta}$, this is not possible.

ClaIm 4.3. There are uniform gradient estimates for $\left\{u_{n}\right\}$ for points in $\alpha_{1} \cup \alpha_{3}$.

Proof. We will use the same idea as in Claim 3.4. For $y_{0}>a$ and $\delta>0$ consider the vertical strip bounded by $\beta_{1}=\left\{\left(x, y_{0}, c_{0}\right): d \leq x \leq d+\delta\right\}, \beta_{2}=\left\{\left(t, y_{0}, c_{0} / d t\right): 0 \leq t \leq\right.$ $d\}, \beta_{3}=\left\{\left(t+\delta, y_{0}, c_{0} / d t\right): 0 \leq t \leq d\right\}$ and $\beta_{4}=\left\{\left(x, y_{0}, 0\right): 0 \leq x \leq \delta\right\}$. This is a minimal surface transversal to the Killing field $\partial_{y}$, hence any small perturbation of its boundary gives a minimal surface with that perturbed boundary. Thus, if we consider a small perturbation of the boundary of this vertical strip by just slightly perturbing $\beta_{1}$ by a curve contained in $\left\{y \leq y_{0}\right\}$ joining the points $\left(d, y_{0}, c_{0}\right)$ and $\left(d+\delta, y_{0}, c_{0}\right)$, we will get a minimal surface $S$ with this perturbed boundary. This minimal surface $S$ will have the property that the tangent planes at the interior points of $\beta_{4}$ are not vertical, by the maximum principle with boundary.

Applying translations along the $x$-axis and $y$-axis, we can use the translates of $S$ to show that $\Sigma_{n}$ is under $S$ in a neighborhood of $\alpha_{3}$, and then we have uniform gradient estimates for points in $\alpha_{3}$. Analogously, constructing similar barriers, we can prove that we have uniform gradient estimates in a neighborhood of $\alpha_{1}$.

Observe that besides the gradient estimates, the translates of the minimal surface $S$ form a barrier for points in a neighborhood of $\alpha_{1} \cup \alpha_{3}$.

We have that $\Sigma_{n}$ is a monotone increasing sequence of minimal graphs with uniform gradient estimates in $\alpha_{1} \cup \alpha_{3}$, and it is a bounded graph for points in $\{x \geq d\}$ (because of the barrier given by the annulus $\mathcal{A}$ ). Therefore, there exists a subsequence of $\Sigma_{n}$ that converges to a minimal surface $\widetilde{\Sigma}$ with $\alpha_{1} \cup \alpha_{3}$ in its boundary. As we already know that $\Sigma_{n}$ converges to the minimal surface $\Sigma$, we conclude that in fact $\Sigma=\widetilde{\Sigma}$, and then $\Sigma$ is a minimal surface with $\alpha_{1} \cup \alpha_{3} \cup \alpha_{4} \cup \alpha_{6}$ in its boundary. Notice that we can assume that $\Sigma$ has $P_{\infty}$ as its boundary, with $\Sigma$ being of class $C^{1}$ up to $P_{\infty} \backslash\{(0,0,0),(0, a, 0)\}$ and continuous up to $P_{\infty}$. The expected 'singly periodic Scherk minimal surface' is obtained by recursively rotating $\Sigma$ by an angle $\pi$ about the vertical and horizontal geodesics in its boundary.

THEOREM 4.4. In any semidirect product $\mathbb{R}^{2} \rtimes_{A} \mathbb{R}$, where $A=\left(\begin{array}{ll}0 & b \\ c & 0\end{array}\right)$, there exists a periodic minimal surface similar to the singly periodic Scherk minimal surface in $\mathbb{R}^{3}$. 


\section{Acknowledgements}

This work is part of the author's PhD thesis at IMPA. The author would like to express her sincere gratitude to her advisor Professor Harold Rosenberg for his constant encouragement and guidance throughout the preparation of this work. The author would also like to thank Joaquín Pérez for helpful conversations about semidirect products.

\section{References}

[1] J. Jost, 'Conformal mappings and the Plateau-Douglas problem in Riemannian manifolds', J. reine angew. Math. 359 (1985), 37-54.

[2] L. Mazet, M. Rodríguez and H. Rosenberg, 'Periodic constant mean curvature surfaces in $\mathbb{H}^{2} \times \mathbb{R}^{\text {', }}$ Asian J. Math., to appear.

[3] W. H. Meeks III, P. Mira, J. Pérez and A. Ros, 'Constant mean curvature spheres in homogeneous three-manifolds', in preparation.

[4] W. H. Meeks III and J. Pérez, 'Constant mean curvature surfaces in metric Lie groups', in: Geometric Analysis: Partial Differential Equations and Surfaces, Contemporary Mathematics, 570 (American Mathematical Society, Providence, RI, 2012), 25-110.

[5] H. Rosenberg, 'Minimal surfaces in $\mathbb{M}^{2} \times \mathbb{R}$ ', Illinois J. Math. 46(4) (2002), 1177-1195.

[6] H. Rosenberg, R. Souam and E. Toubiana, 'General curvature estimates for stable $H$-surfaces in 3-manifolds and applications', J. Differential Geom. 84(3) (2010), 623-648.

\footnotetext{
ANA MENEZES, Instituto Nacional de Matemática Pura e Aplicada (IMPA), Estrada Dona Castorina 110, 22460-320, Rio de Janeiro-RJ, Brazil

e-mail: anamaria@impa.br
} 